
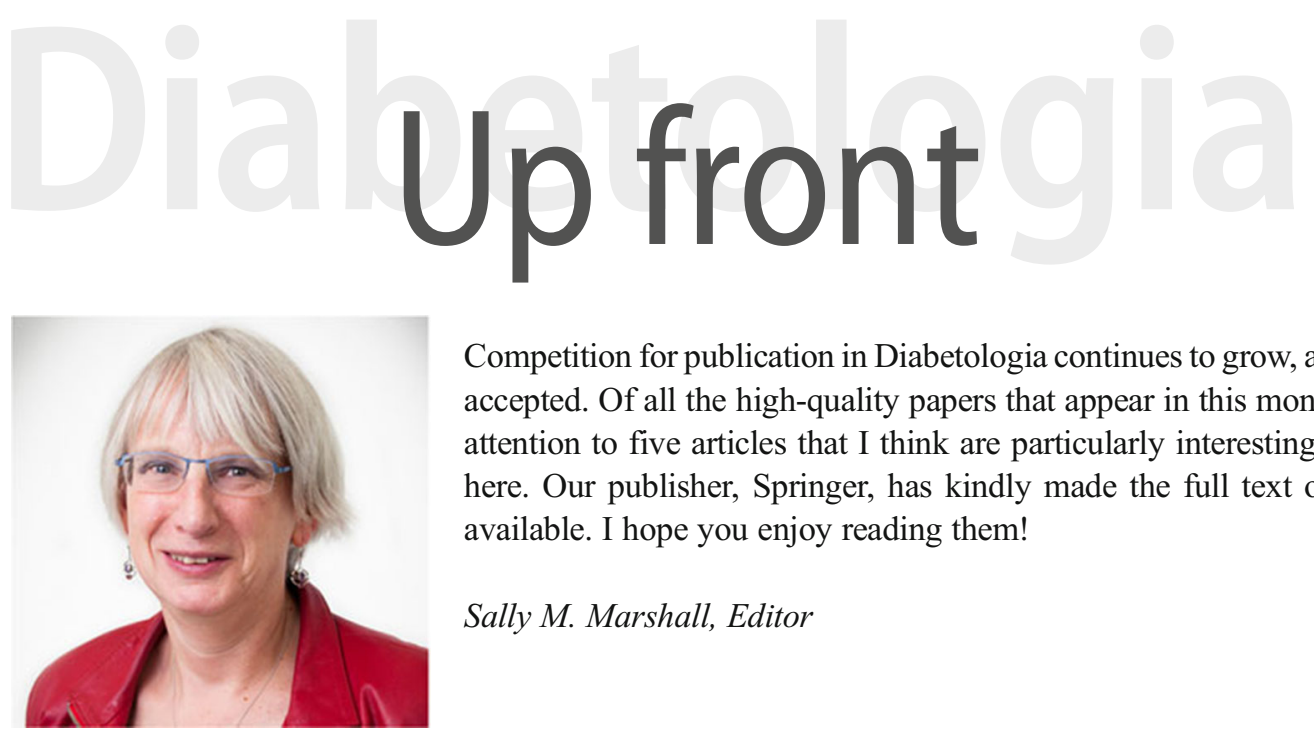

Competition for publication in Diabetologia continues to grow, and less than $20 \%$ of papers are accepted. Of all the high-quality papers that appear in this month's issue I want to draw your attention to five articles that I think are particularly interesting. The articles are summarised here. Our publisher, Springer, has kindly made the full text of each of these papers freely available. I hope you enjoy reading them!

Sally M. Marshall, Editor

\section{Epidemiology of diabetes and diabetic complications in China}

Ronald C. W. Ma

Over the last few decades, China has witnessed one of the most dramatic increases in diabetes prevalence and currently has the largest population with diabetes in the world. In this issue, Ronald Ma (https://doi.org/10.1007/s00125-018-4557-7) summarises recent updates on the diabetes epidemic in China. He notes the increasing proportion of individuals with young-onset diabetes in China, as well as the increasing prevalence of gestational diabetes, with the potential for intergenerational effects on offspring. Cardiovascular and renal complications remain major causes of death in individuals with diabetes, though cancer and dementia are being increasingly recognised as important comorbidities of diabetes. Structured assessment and care delivery has been associated with improved risk factor control and reduced complications in some regions, such as Hong Kong. However, there remain significant challenges and notable treatment gaps, and multi-pronged strategies have been implemented to tackle these. The author highlights that prevention and optimal management will require good medical care, as well as improved health literacy, patient empowerment and a health-enabling environment.

(D) The figures from this review are available as a downloadable slideset.
Extracellular matrix molecules and their potential contribution to the function of transplanted pancreatic islets

L. Alberto Llacua, Marijke M. Faas, Paul de Vos

Understanding the nature of the extracellular matrix (ECM) and how it interacts with resident cells is the next step towards the recreation of biochemical cues for directing cell function in an artificial organ. Although transplantation of pancreatic islets has shown good clinical efficacy in patients with type 1 diabetes, the sudden loss of the pancreatic environment, subsequent interruption of cell-matrix interactions by isolation enzymes, and the lack of treatment to restore the ECM negatively impacts functional survival of pancreatic islet grafts. In this issue, Llacua et al (https://doi.org/10.1007/s00125-0174524-8) summarise recent advances in our understanding of pancreatic islet cell membrane biology, specifically discussing the potential contribution of major ECM components to the function of transplanted pancreatic islets. The authors propose that developing knowledge of the crucial role the ECM plays in maintaining and driving endocrine function may facilitate the construction of supplemented bioartificial scaffolds to support islet function and improve glucose responsiveness and islet survival, advancing this therapeutic avenue for diabetes.

(D) The figures from this review are available as a downloadable slideset. 
Metabolomics insights into early type 2 diabetes pathogenesis and detection in individuals with normal fasting glucose

Jordi Merino, Aaron Leong, Ching-Ti Liu, Bianca Porneala, Geoffrey A. Walford, Marcin von Grotthuss, Thomas J. Wang, Jason Flannick, Josée Dupuis, Daniel Levy, Robert E. Gerszten, Jose C. Florez, James B. Meigs

Given the worldwide diabetes epidemic, it is important to evaluate novel approaches to improve type 2 diabetes prediction. This would allow us to identify people at elevated risk, who may benefit from focused prevention interventions. Prior studies have identified plasma metabolites which are associated with the development of type 2 diabetes among individuals initially without diabetes. However, the measurement of these metabolites does not greatly improve prediction of new diabetes beyond common clinical risk factors, such as BMI. In this issue, Merino et al (https://doi.org/10.1007/s00125-0184599-x) report that metabolomic differences existed in individuals with normal blood glucose levels at baseline who developed diabetes over 20 years of follow-up, compared with individuals who remained diabetes free. Specifically, 19 metabolites were associated with type 2 diabetes incidence and measurement of these metabolites substantially improved prediction of future diabetes beyond common clinical risk factors. The authors propose that, if these findings are confirmed in other populations, we now have evidence that measurement of a defined set of metabolites could help identify individuals at risk of future type 2 diabetes, even when they appear clinically at low risk.

\section{Angptl8 antisense oligonucleotide improves adipose lipid metabolism and prevents diet-induced NAFLD and hepat- ic insulin resistance in rodents}

Daniel F. Vatner, Leigh Goedeke, Joao-Paulo G, Camporez, Kun Lyu, Ali R. Nasiri, Dongyan Zhang, Sanjay Bhanot, Susan F. Murray, Christopher D. Still, Glenn S. Gerhard, Gerald I. Shulman, Varman T. Samuel

Obese individuals do not all develop the same metabolic complications: some are metabolically healthy, while others develop insulin resistance, fatty liver and atherosclerosis. These metabolic complications are associated with ectopic lipid accumulation (e.g. in the liver, skeletal muscle) and, thus, improving adipose lipid storage could be beneficial. One possible ap- proach is to enhance adipose lipoprotein lipase (LPL) activity, as this enzyme regulates tissue lipid uptake. In this issue, Vatner et al (https://doi.org/10.1007/s00125-018-4579-1) report that adipose tissue expression of $A N G P T L 8$, an inhibitor of LPL, is higher in obese individuals with metabolic complications (insulin resistance and fatty liver) than in BMI-matched insulin-sensitive individuals. Decreasing angiopoietin-like 8 (ANGPTL8) expression using a synthetic Angptl8 antisense oligonucleotide in fat-fed rodents improved the clearance of meal-derived lipid molecules by adipose cells and, in turn, decreased the deposition of lipids in the liver and improved insulin sensitivity. These findings suggest that endogenous LPL inhibitors, such as ANGPTL8, could prove to be valuable targets to treat the metabolic complications of obesity. This article and the article by Janssen et al (see below) are the subject of a commentary in this issue by Brandon Davies (https://doi.org/10.1007/s00125-018-4604-4).

Loss of angiopoietin-like 4 (ANGPTL4) in mice with dietinduced obesity uncouples visceral obesity from glucose intolerance partly via the gut microbiota

Aafke W. F. Janssen, Saeed Katiraei, Barbara Bartosinska, Daniel Eberhard, Ko Willems van Dijk, Sander Kersten

The protein angiopoietin-like 4 (ANGPTL4) is known to reduce fat storage and raise circulating triacylglycerol levels by inhibiting the enzyme lipoprotein lipase (LPL). However, the role of ANGPTL4 in glucose homeostasis is less clear. In this issue, Janssen et al (https://doi.org/10.1007/s00125-0184583-5) report that, despite having increased visceral adipose tissue mass and LPL activity, mice lacking ANGPTL4 (Angptl4 ${ }^{-1-}$ mice) have markedly improved glucose tolerance accompanied by elevated insulin levels compared with wild-type mice. Interestingly, suppression of the gut microbiota using antibiotics largely eliminated the differences in glucose tolerance and insulin levels between wildtype and Angptl4 ${ }^{-1-}$ mice. These findings indicate that ANGPTL4 uncouples the link between reduced adiposity and improved glucose tolerance, at least partly via a gut microbiota-dependent mechanism. The authors conclude that targeting ANGPTL4 might be therapeutically relevant beyond the lowering of plasma triacylglycerol levels. This article and the article by Vatner et al (see above) are the subject of a commentary in this issue by Brandon Davies (https://doi.org/ 10.1007/s00125-018-4604-4).

All text supplied by the authors. 